incisions taking with it the iris and probably necessitating further surgical interference in the form of an iridectomy. On the other hand once the conjunctival flap has healed, there is sufficient back pressure on the aqueous in the anterior chamber to prevent its sudden escape through the triangular opening, there is now no danger of the iris prolapsing.

As soon as massage has been started it may be carried out once or twice daily during the post-operative period to ensure the passage of aqueous through the opening and to prevent healing occurring at its edges. The patient is allowed up at this juncture and can usually be discharged around the tenth day. He is instructed to massage the eyeball gently daily through the lower lid and is seen at weekly. intervals until it has become obvious that a permanent filtering cicatrix has been produced; at this stage massage is discontinued.

\title{
OPHTHALMO-NEUROLOGICAL SYMPTOMS IN CONNECTION WITH MALIGNANT NASOPHARYNGEAL TUMOURS*
}

BY

\author{
DR. ERIK GODTFREDSEN
}

COPENHAGEN

\begin{abstract}
From Radiumhemmet, Stockholm (Professor L. Berven), Kung Gustaf $V^{\prime}$ tes Jubilaumsklinik, Lund (Professor L. Edling), and the Radiological Clinics of Copenhagen (Physician-in-Chief, Jans Nielsen) and Aarhus (Professor C. Krebs).
\end{abstract}

Mr. Chairman, Ladies and Gentlemen,

I feel greatly pleased and honoured to be allowed to speak before this dignified audience and thus to re-establish the connection between British and Danish ophthalmology, a connection which was previously so intimate and profitable. Danish ophthalmologists, among whom the best known are Bjerrum, Tscherning, Edmund Jensen, Heerfordt, Lundsgaard, and Roenne, are greatly indebted to British ophthalmologists, whose best known representatives with us are men like Young, Mackenzie, Bowman, Priestley-Smith, Marcus Gunn and Duke-Elder.

During the gloomy years of German occupation Danish ophthalmology greatly missed the inspiration from British ophthalmology. Now that I stand here as the first Danish speaker after the war I

* Read before the Ophthalmic Section, Royal Society of Medicine, London, November 14, 1946. + Teceived for publication, December 6, 1946. 


\section{OPhTHALMO-NeURological SYMPTOMS in ConNection With Malignant Nasopharyngeal TUMOURS}

naturally call to mind the heroic contribution made by the English towards the liberation of my native country, for which we are all very grateful, and this will never be forgotten.

Realising the importance for us in Denmark of the re-establishment of this connection I thank you for your kind invitation to the present meeting, and I hope I shall not betray the trust reposed upon me by the Society.

As the subject of which I want to speak has been dealt with but rarely in British literature, I hope to be able to bring forward for discussion facts which are little known to you.

\section{Introduction}

An account of the ophthalmo-neurological symptoms in connection with malignant nasopharyngeal tumours must necessarily include a description of other important features of the symptomatology to give the right idea of the position in the nosography of the ophthalmo-neurological symptoms.

An increased interest, though varying in degree, has been taken in malignant nasopharyngeal tumours since the introduction, about 100 years ago, of the postrhinoscopic speculum examination, through which it has become possible to diagnose diseases in the nasopharynx. This interest has been further stimulated within the past few decades on account of the results obtained from modern radiological treatment, which to-day is capable of curing these deep-seated and generally inoperable tumour forms. The increased interest is, however, due to other facts as well, thus for instance to the fact that these tumour forms have proved to be of more frequent occurrence than they were previously supposed to be, and that the disease is very often misjudged.

The cause of this misjudgment may to a certain extent be explained from the variety of the clinical picture, which but rarely conveys the idea of a malignant neoplasm in the nasopharynx. That a. no small number of patients are young persons often in sound general health likewise contributes to this fact.

The widely different symptoms or combinations of symptoms imply, besides applications to general practitioners, also examinations of the patients by various specialists often at rather early stages, thus by ophthalmologists, neurologists, oto-rhinologists, surgeons, neurosurgeons, internists and radiologists.

If the idea of a malignant nasopharyngeal tumour in the case in hand does not occur to the examining physician and the time for the making of the right diagnosis is delayed, by which valuable-perhaps life-saving - time is wasted for the patient. 


\section{Historical data}

I shall abstain from a detailed historical review of the literature in hand. Only I want to mention that the nosographical pioneer works are due chiefly to French writers (Escat, Laval, Jacod), and in England to Trotter, while most of the modern comprehensive descriptions come from America (New and others).

Nasopharyngeal tumours were the order of the day at the International Medical Congress in London; 1913. But very little was said about ophthalmological symptoms.

\section{Frequency, histopathology, age and sex incidence}

The malignant nasopharyngeal tumours, though of somewhat infrequent occurrence are by no means rare. Thus over 2,000 cases have now been published in thefliterature, and personally I managed to collect 454 cases over a period of ten years from the four Scandinavian radiological clinics. The following numerical statements are based on these latter cases.

The four radiological clinics from which the cases under review are derived are as follows: Radiumhemmet, Stockholm and the Radiological Clinics of Lund, Copenhagen, and Aarhus, which together cover a population corresponding in size to that of London (about $7 \frac{1}{2}$ million people). The frequency of malignant nasopharyngeal tumours constituted here 1 per cent. of the total number of cancer cases, i.e., a frequency of the same relative magnitude as that of choroidal melanosarcoma. As, however, only the cancer cases that are likely to respond to irradiation are referred to the radiological clinics, we must make a correction for this percentage to attain to the frequency among the total number of cancer cases in the population. The figure then arrived at is 0.4 per cent. of all cases of cancer.

A bare two-fifths (38 per cent.) of the patients presented ophthalmo-neurological symptoms, a figure which varied but little from one year to the other. -

Histopathological examinations revealed both carcinomata and sarcomata, in conformity with the fact that malignant nasopharyngeal tumours proceed from the mucous membrane and the lymphatic tissue in the nasopharynx. I shall not go into details regarding these facts, but only just mention that the majority of the tumours were anaplastic tumours of low differentiation. Most common were squamous epithelial carcinomata and reticulum-cell sarcomata, which occurred equally often. The undifferentiated tumours and those of low differentiation constituted four-fifths of the cases, while the differentiated tumours, i.e., squamous epithelial carcinomata undergoing cornification constituted one-fifth.

As to the age incidence (Fig. 1) all age-classes from 4 to 79 were represented, but half of the patients were found within the age-classes of 41 to 60 . It is, however, worth noting that one-fourth of the patients were 40 years of age or younger. The average age was 49 . When cases of carcinoma and sarcoma were taken separately the average age was somewhat higher for carcinomata than for
sarcomata.

For no discoverable reason there were twice as many men as women both among the carcinoma cases and among the sarcoma cases. 


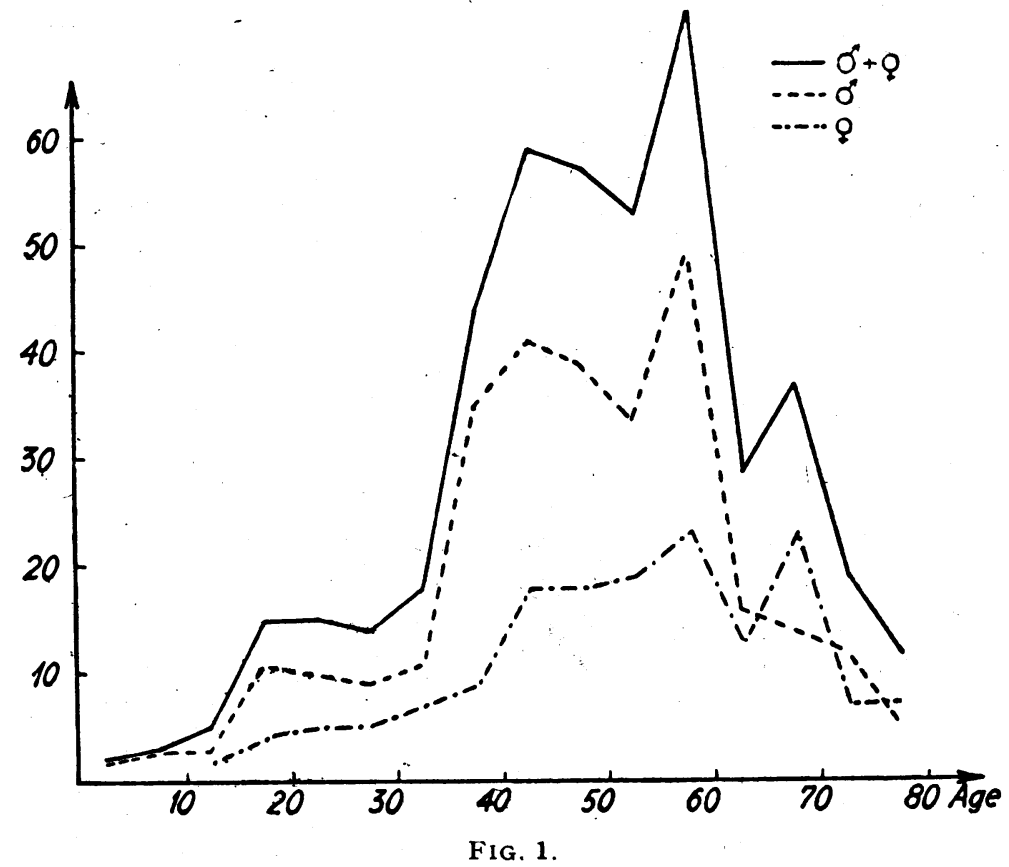

Age-curves for 454 cases of malignant nasopharyngeal tumours.

\section{Symptomatology}

The symptomatology can roughly be divided into four wellcharacterised groups :

(1) Ophthalmo-neurological symptoms due to the infiltrative growth of the primary tumour or its metastases into the base of the skull and adjacent regions,

(2) rhinological and

(3) otological symptoms due to the exophytic growth of the primary tumour into the nasopharynx with influence on the Eustachian tube,

(4) enlarged cervical glands in consequence of lymphogenous metastases.

I shall here give a brief survey of the manner of growth of the tumours and of the topographical anatomy of the affected areas, because the understanding of the symptomatology of the malignant nasopharyngeal tumours, in particular the ophthalmo-neurological symptomatology, rests to a great extent on a knowledge of these facts.

The most frequent point of origin of the primary tumour is the lateral wall of the nasopharynx, especially the lymphatic tissue of the tubal tonsil (half of the cases). Next in frequency follows the 
roof (the nasopharyngeal tonsil, one-third of the cases), and finally the posterior wall (Fig. 2).

A characteristic feature of the malignant nasopharyngeal tumours is their tendency to infiltrative and destructive penetration

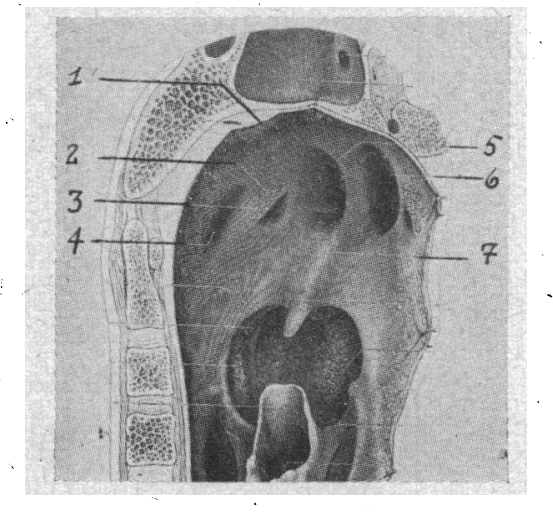

FIG. 2.

The nasopharynx seen from behind and from the right side with the characteristic relief: pharyngeal tonsil (1), tubal tonsil (2), tubal ostium (3), Rosenmüller's pharyngeal recess (4). The soft palate (7) and the posterior border. of the nasal septum (5) bound the choanae (6). (After Testut).

into the adjacent areas, partly the parapharyngeal space and partly the base of the skull. The tumour either follows the foramina already present or grows with osseous perforation. The parts of the base that are particularly exposed to invasion are (Fig. 3)

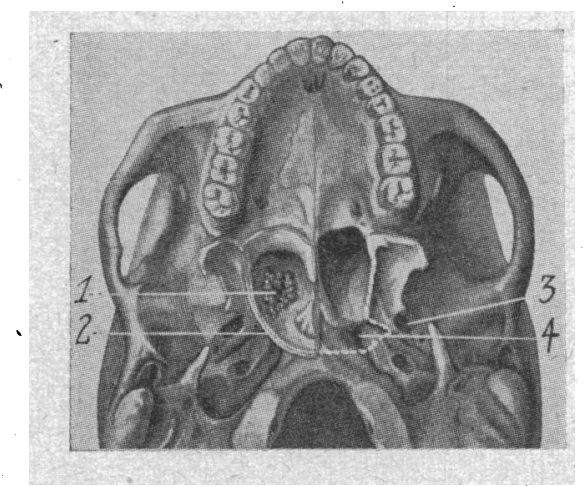

FIG. 3.

The limit of the nasopharynx on the external base of the skull appears from the dotted white line running between the foramen lacerum (4) and the foramen ovale (3). To the left in the picture the nasopharyngeal wall is drawn (2) with a neoplasm arising from the tubal ostium (1). (The Fig. modified after Canuyt). 
naturally those which lie along the line of demarcation between nasopharynx and base of the skull, which is marked by the place of insertion of the pharyngo-basilar fascia. This area is seen to

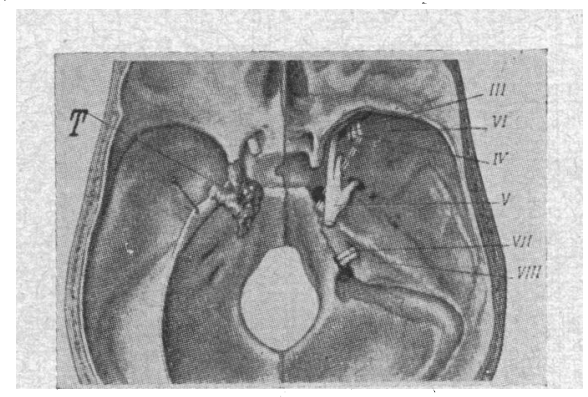

FIG. 4.

Internal base of the skull. The Roman numerals indicate the numbers of the brain nerves. To the left (T) there is seen a nasopharyngeal tumour invading the middle cranial fossa through the foramen lacerum (Canuyt).

comprise parts of the body of the sphenoid bone, the basilar portion of the occipital bone, as well as the apex of the pars petrosa. These three osseous portions encompass the foramen lacerum. When the tumour tissue grows up through the foramen lacerum it reaches the internal base of the skull in the middle cranial fossa (Fig. 4) with intimate relation to the cavernous sinus and the cranial nerves situated in this place, that is III, IV, V, and VI (Fig. 5). Pressure or direct penetration into the nerves will then bring about increasingly severe lesions particularly of the abducens

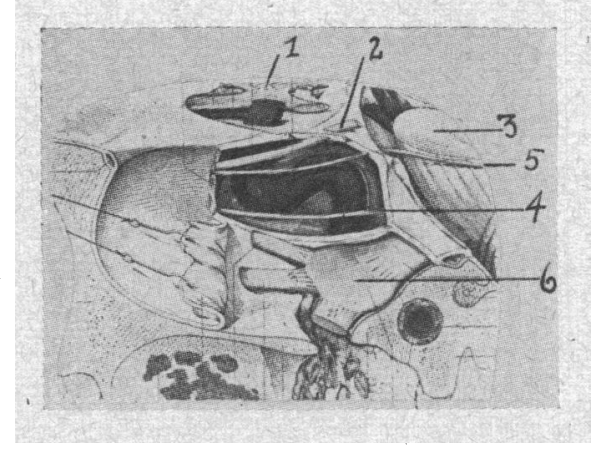

FiG. 5.

Left middle cranial fossa with the cavernous sinus open, seen from the side. Superiorly the optic chiasma (1) and the third nerve (2), under which the thin fourth nerve (5) running along the mesencephalon (3). Further the sixth nerve (4) and the Gasserian ganglion (6) with the ramifications (after Testut). 
and the trigeminus, resulting in eye muscle paralyses and varying degrees of trigeminal neuralgias or disturbances of sensibility, and possibly paralysis of the muscles of mastication (Figs. 6 and 9).

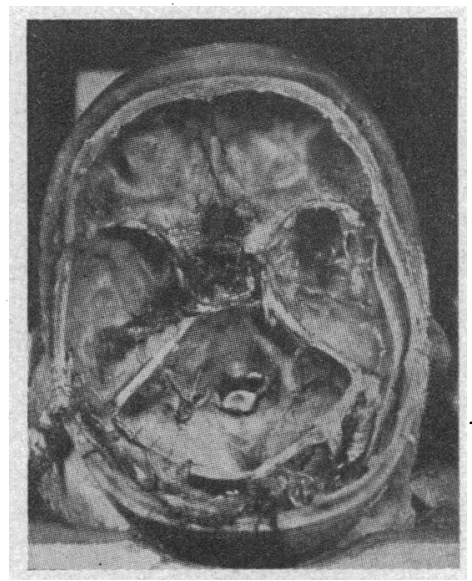

Fig. 6.

Internal base of the skull of a female, aged 29 years (case 141), presenting a large tumour invasion through the body of the sphenoid bone (destruction of sella and pituitary) and left cavernous sinus from a nasopharyngeal reticulum-cell sarcoma. Clinically there was found left ophthalmoplegia and amaurosis.

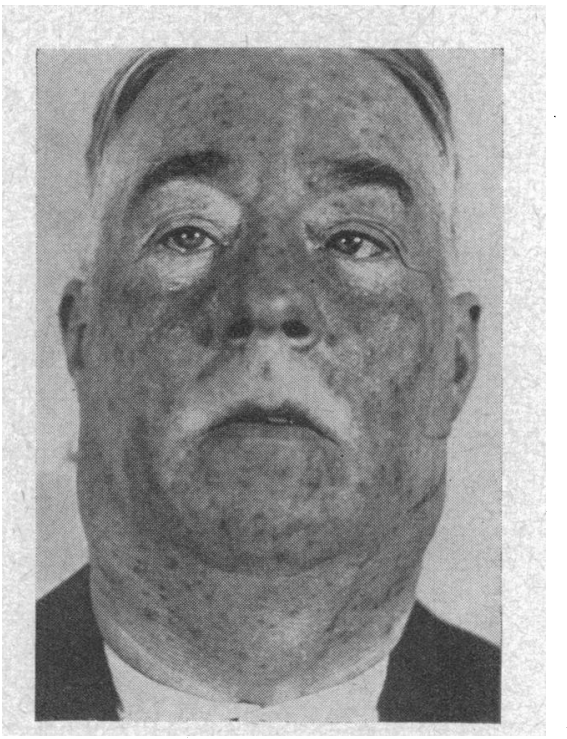

Fig. 7.

Male, aged 56 years (case 81), with left paralysis of the sixth cranial nerve (of one month's duration) and bilateral cervical glands from squamous cell carcinomas on left Eustachian tube. 
OPHTHALMO-NEURological SyMPTOMS in CONNECTION WITH MALIGNANT NASOPHARYNGEAL TUMOURS

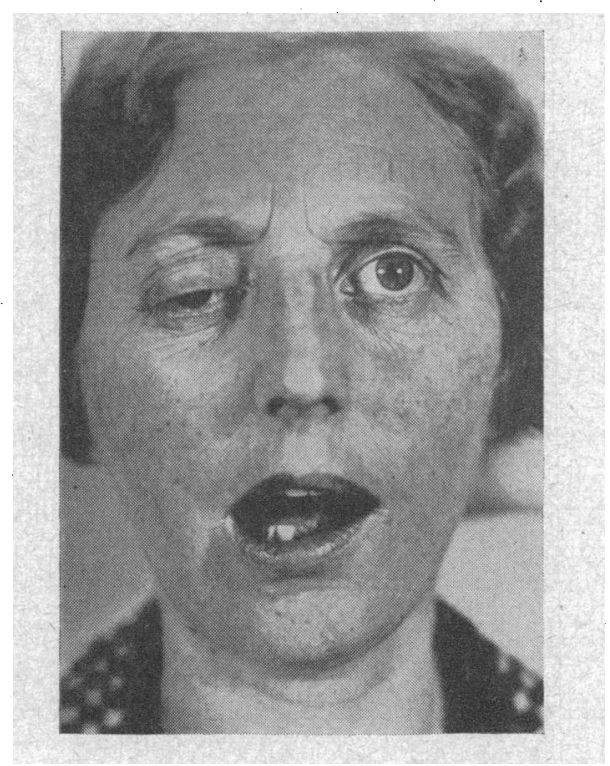

FIG. 8.

Female, aged 40 years (case 83 ) with right paralysis of the third cranial nerve (of two month's duration). No metastatic cervical glands. Destruction of the base of the skull verified by $\mathrm{X}$-rays. In the nasopharynx a reticulum cell sarcoma proceeding from right tube.

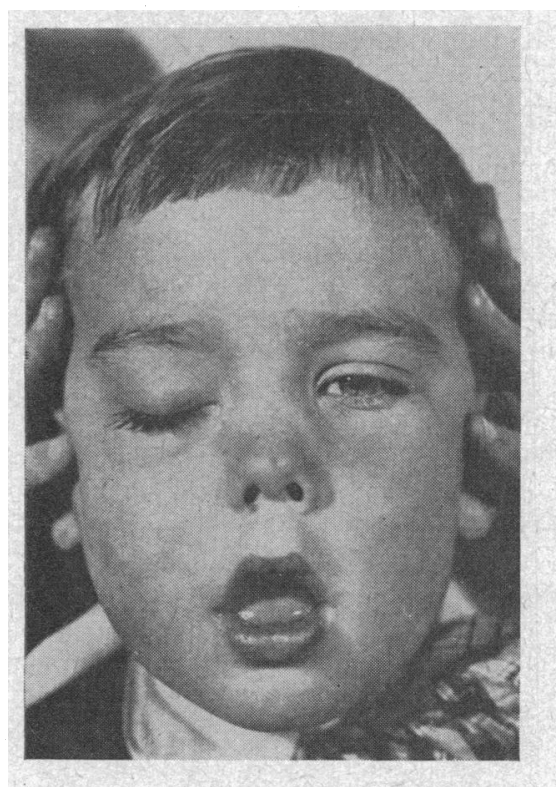

F1G. 9.

Boy, aged 5 years (case 84) with right total ophthalmoplegia (developed through two months) and nasal stenosis (of five month's duration) as well as right cervical glards (of one year's duration) from malignant nasopharyngeal tumour, presumably reticulum-cell sarcoma. 
If the tumour grows upwards and forwards the visual pathway will be injured, and this will result in visual disturbances in the forms of impairment of vision, defects of the field of vision, and different ophthalmoscopical findings, such as choked disc or atrophy.

In cases where the tumour penetrates through the superior orbital fissurethere will occur exophthalmos (Fig. 10), which may, however,

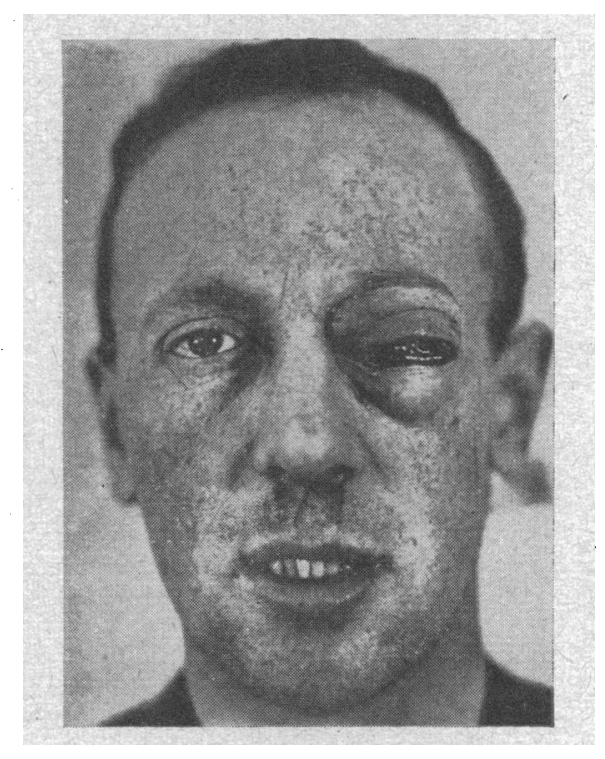

'Fig. 10.

Male, aged 24 years (case 30 ) with excessive left exophthalmos and chemosis developed in the course of two months by a reticulum cell sarcoma from the nasopharynx invading the orbit. Cervical gland metastasis the size of a hazel nut (left).

also be due to orbital invasion through the inferior orbital, fissure through the parapharyngeal space.

The simultaneous occurrence of ophthalmoplegia, optic tract lesion with amaurosis, and trigeminal neuralgia has been described by Jacod as syndrome carrefour petrosphénoidale and is regarded as pathognomonic of malignant nasopharyngeal tumours.

Penetration of the tumour into the parapharyngeal space in the direction towards the jugular foramen and the hypoglossal canal will bring about different radial symptoms from the cranial nerves running in this area, i.e., $\mathrm{IX}, \mathrm{X}, \mathrm{XI}$, and $\mathrm{XII}$, with paraesthesias and paralyses of pharynx, palate, larynx, and tongue.

The truncus sympatheticus can be injured partly in this place 


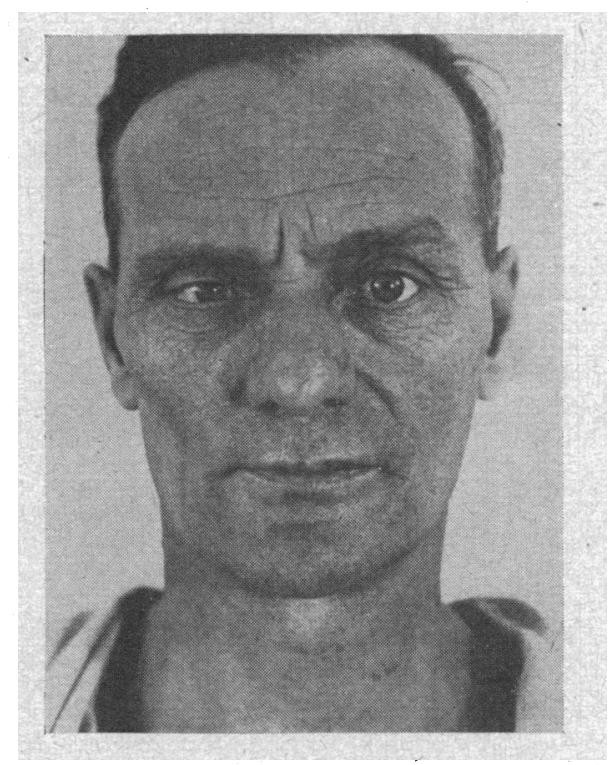

Fig. 11.

Male, aged 42 years (case 69) with ipsilateral (right) Horner's syndrome (of six month's duration), paralysis of the sixth cranial nerve (of two month's duration, and cervical glands (of three month's duration) from reticulum-cell sarcoma proceeding from the tube (presumably).

and partly intracranially (paratrigeminally), or in its cervical portion with the development of Horner's.syndrome (Fig. 11).

Paresis of the seventh cranial nerve, with or without attending eighth nerve lesion, may occur by growth of the tumour in the direction towards the porus acousticus internus; but more often the seventh cranial nerve is injured about its point of exit from the facial canal at the stylomastoid foramen.

All these symptoms are at their initial stages due to pressure from the tumour tissue, but later also to irreversible destructions. That is the reason why nervous symptoms are often seen to improve after irradiation at the initial stages.

Since these ophthalmo-neurological symptoms may be the first and only symptoms of a nasopharyngeal tumour, it is no wonder that such cases are often mistakenly diagnosed and treated symptomatically for eye muscle paralyses or trigeminal neuralgia for a considerable length of time without the slightest effect.

The rhinological and otological symptoms in connection with malignant nasopharyngeal tumours are, as mentioned already, due to the exophytic, space-filling growth of the tumour, and also to the tendency of the tumour to ulceration and to development 
of stenosis of the air passage, partly through the nose and partly through the Eustachian tube.

The purely rhinological symptoms are thus: uni- or bilaterally reduced or arrested nasal respiration, as well as nasal discharge and epistaxis.

The influence of the tumour on the Eustachian tube is sometimes due to mechanical compression and sometimes to penetration of the tumour into the tube. The result is in both cases tubal occlusion, which may be intermittent at first, but will soon become permanent, and manifests itself by tinnitus, clicking sensation, and impairment of hearing. The tubal occlusion involves a vacuum in the middle ear with pains in the ear and consecutive exudation, which at secondary infection gives a picture resembling that of otitis media.

Unfortunately the nasal and otogenous complaints are often misjudged, so that the patients are submitted to different Qperations of the skeletal and soft tissues in the nose, tubal catheterisations; paracenteses, etc. The frequent mistaken diagnoses are probably due to the fact that the primary tumour is often diminutive and conscquently difficult to recognise for untrained examiners.

The fourth and last main group of signs and symptoms is that of enlarged cervical glands, which are due to the pronounced tendency of the tumour to early development of lymphogenous metastases, which can reach rather considerable degrees even bilaterally, even when the primary tumours are diminutive.

The typical glandular metastases develop posterior to the angle of the jaw, over the mastoid process beneath the sterno-mastoid, whence they later extend distally. This accords well with the

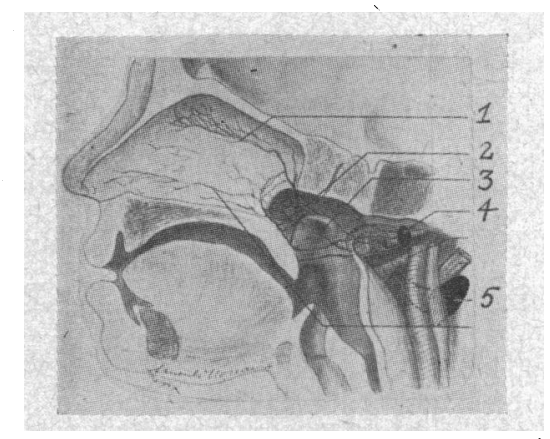

FIG. 12.

Sagittal section of the head seen from within (after Rouviere) with the lymph stems from the nasal septum (1) the lateral wall of the nasopharynx (2) and the tubal ostium (3) to the lateral retropharyngeal lymph node (4) and the superior deep cervical lymph nodes (5).

course of the lymph from the nasopharynx, which passes by the retropharyngeal lymph glands-or direct-to the superior group of the deep cervical lymph glands (Fig. 12). Clinically there occurs (Figs. 13, 14, 15) a steadily increasing, indolent node, whose consistency and fixation to the surrounding tissue depend on the type of the primary tumour, but is generally, however, rather firm and fixed deeply. 


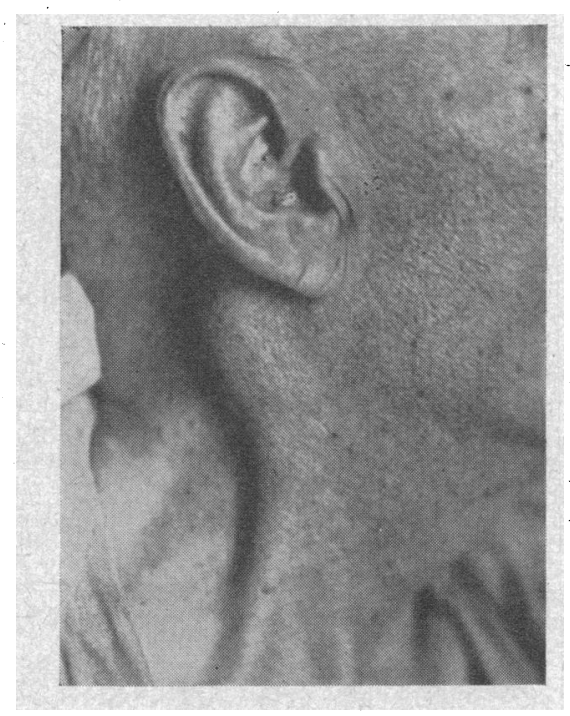

FIG. 13.

Male, aged 70 years (case 376) with right cervical gland metastasis in the place of election (developed through nine months) from as quamous cell carcinoma in the nasopharynx.

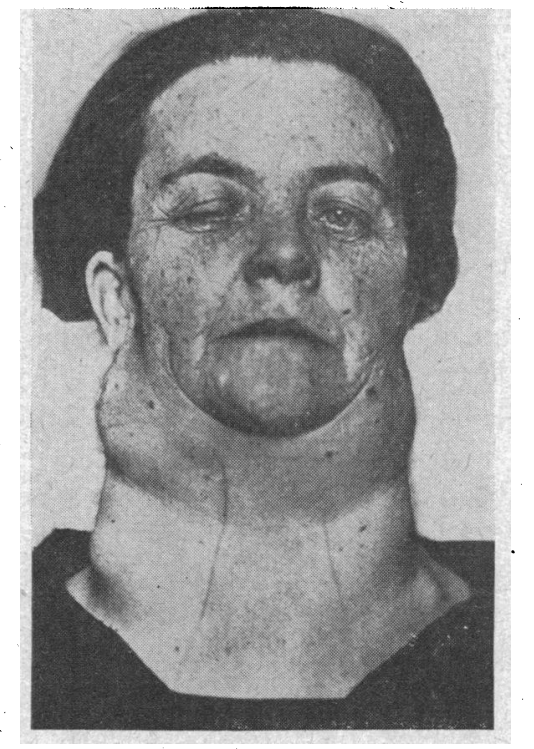

F1G. 14.

Female, aged 50 years (case 1) with bilateral paralysis of the sixth cranial nerve and right paralysis of the third nerve. Large bilateral cervical glands (of one year's duration) from squamous cell carcinoma on right nasopharyngeal wall. 


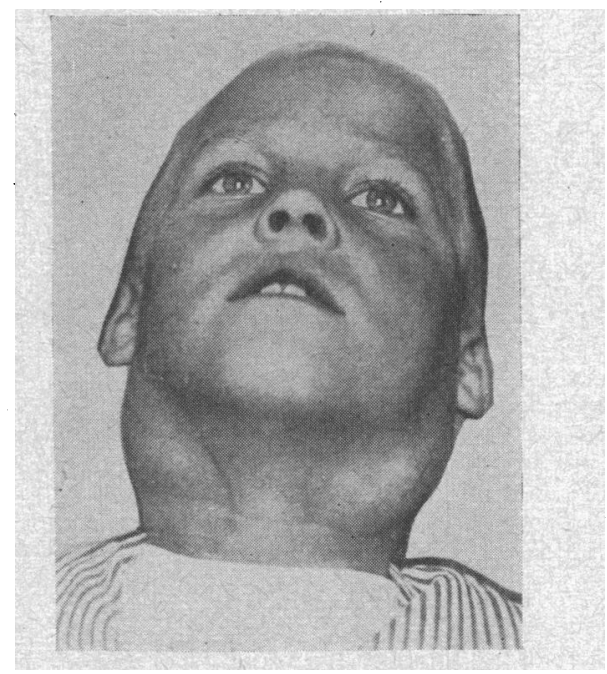

FiG. 15

Boy, aged 10 years (case 205) with large bilateral cervical gland metastases (of 12 month's duration, regarded as tuberculosis lymphomas. Treated in seaside health resort, etc.), from a large lymphocytosarcoma in the nasopharynx.

The cervical glands being frequently the only sign of a nasopharyngeal tumour through half or whole years they are often mistakenly diagnosed as lymphomas of tuberculous or other infectious origin and treated by light baths, incisions, light $\mathrm{X}$-rays, etc.

The total number of mistaken treatments for all the groups of symptoms amounts to at least one-third of all the patients.

\section{Syptomatology of malignant nasopharyngeal tumours elucidated from own examination of 454 patients}

After this more general review of the symptomatology I shall now pass on to a more statistically based account of the different symptoms.

As it is urgently necessary, in cases of malignant nasopharyngeal tumours, to have the diagnoses made both earlier and with. greater certainty than is now the case, it is of importance to concentrate particularly on an elucidation of the early symptoms of these tumour forms. To give as clear a picture as possible of the often very polymorphous course of the tumours I have chosen to make an analysis of the symptomatology at three differcnt stages of the disease.

The first analysis covers the first symptom(s) observed by the 
patient. It occurs on an average 11 months (3 or 4 years at most) before the exact diagnosis is made.

The second analysis covers the initial symptomatological picture in a wider sense. At this stage there are found several symptoms within one group, or different groups are represented. This pathological picture is seen on an average 4 or 5 months before the exact diagnosis is made.

The third analysis covers the fully developed picture, which corresponds to the point of time at which the diagnosis is made and the patient referred to a radiological clinic.

The percentage frequency of ophthalmo-neurological symptoms at these three stages appears from Table 1, into which also the frequencies of the three other main groups of symptoms have been entered. The stated percentage figures refer-with inconsiderable limitations-to the 454 cases from my M.D. thesis.

Table 1 shows that ophthalmo-neurological complaints are the first symptom in 16.0 per cent. of the cases, while metastatic cervical glands and rhinological symptoms are both present as the first symptom in about one-third of the patients. Otological

\section{TABLE I.}

Percentage distribution of the different groups of signs and symptoms at three stages of the disease among 454 cases of malignant nasopharyngeal tumour.

\begin{tabular}{|c|c|c|c|}
\hline . & $\mid \begin{array}{c}1 \text { st symptom } \\
11 \text { months } \\
\text { before diag- } \\
\text { nosis is made }\end{array}$ & $\begin{array}{l}\text { Initial picture } \\
4 \text { or } 5 \text { months } \\
\text { before diag- } \\
\text { nosis is made }\end{array}$ & $\begin{array}{c}\text { Fully deve- } \\
\text { loped picture } \\
\text { when the } \\
\text { diagnosis is } \\
\text { made }\end{array}$ \\
\hline Ophthalmo-neurological symptoms & $16^{\circ} 0$ & $34^{\circ} 8$ & $38^{\circ} 0$ \\
\hline Rhinological symptoms $\ldots$ & $30^{\circ} 8$ & 51.4 & $56^{\circ} 2$ \\
\hline Otological symptoms & $23 \cdot 3$ & $48 \cdot 5$ & $49^{\circ} 1$ \\
\hline Metastatic cervical glands ... & $32 \%$ & $4.8^{\circ} 4$ & $74^{\circ} 9$ \\
\hline
\end{tabular}

symptoms are rarer, being present in about one-fifth of the cases only. Nearly all the symptoms are monosymptomatic at this stage of the disease (in nine-tenths of the cases); and it must be emphasised that two-thirds of the patients did not complain of nasal symptoms, and that metastatic cervical glands were present already by this time in 32.0 per cent. of the cases.

At the next stage of the analysis, that is 4 or 5 months before the exact diagnosis is made, the most conspicuous changes are 
as follows : The ophthalmo-neurological symptoms have more than doubled in frequency occurring now in 34.5 per cent., whereas the other groups of symptoms present more moderate increases in frequency, each being found in half of the patients. The frequency of monosymptomatic cases has become reduced to comprising onefourth of the cases only against nine-tenths at the first stage.

At the third and final stage of the analysis-by the time the diagnosis is made-there are two facts which are particularly noteworthy: First the frequency of ophthalmo-neurological symptoms is rather stationary, being found in a total of two-fifths of the cases (or 38.0 per cent.), and secondly there is a considerable increase in the frequency of metastatic cervical glands, which are now present in three-fourths of the patients. Rhinological and otological symptoms, on the other hand, are rather stationary in frequency, occurring in about half of the cases.

These analyses show plainly that when ophthalmo-neurological symptoms do occur in cases of malignant nasopharyngeal tumour they manifest themselves at early stages. The ophthalmoneurological cases can be said to have been separated out as a special group already on an average 4 or 5 months before the exact diagnosis is made. In other words, these symptoms do not -as previously believed-occur only at the advanced and hopeless stages of the disease, a fact which must stimulate our interest in a more energetic tracing of these cases.

The fact that the group of metastases in the cervical glands increases so considerably in frequency from the initial to the fully developed symptomatic picture is a serious reminder of the biological activity and tendency to spreading of these tumour forms. This again means that if these cases can be diagnosed but a fer months earlier than is now the case, then one of the consequences will be that the patients will be submitted to irradiation before the metastases have spread too far. The chance of obtaining a favourable result from treatment with $\mathrm{X}$-rays will then be increased very considerably.

Thus there appear to be various reasons for stimulating the interest in and knowledge of the initial symptomatology of the malignant nasopharyngeal tumours, in particular the ophthalmoneurological symptoms.

\section{Nature and frequency of ophthalmo-neurological symptoms}

After this statement of the place in the symptomatology of the ophthalmo-neurological symptoms at the different stages of the disease, I shall now pass on to a more detailed description of these symptoms.

Be it stated at once that all forms of cranial nerve lesions are met with, as might have been expected from the manner of growth of 
the tumour, but that there is found a certain systematisation. Thus for instance lesions of eye nerves and/or trigeminus are found in practically all cases. Bęfore entering on a more detailed account I shall just mention an important proportional change in frequency between eye nerve lesions and trigeminus lesions at the three stages of the disease, where analyses have been made.

TABLE II.

Increase in frequency (per cent.) of ophthalmological symptoms compared with that of trigeminus lesions-from the first symptom to the fully developed picture.

\begin{tabular}{|c|c|c|c|}
\hline$\cdot$ & $\begin{array}{c}\text { 1st symptom } \\
11 \text { months } \\
\text { before diag- } \\
\text { nosis is made }\end{array}$ & $\begin{array}{l}\text { Initial picture } \\
4 \text { or } 5 \text { months } \\
\text { before diag- } \\
\text { nosis is made }\end{array}$ & $\begin{array}{c}\text { Fully deve- } \\
\text { loped picture } \\
\text { when the } \\
\text { diagnosis is } \\
\text { made }\end{array}$ \\
\hline $\begin{array}{l}\text { Frequency of ophthalmological } \\
\text { sympt. (per cent.) } \ldots\end{array}$ & $14^{\circ} 4$ & $51^{\circ} 0$ & $75^{\circ} 2$ \\
\hline 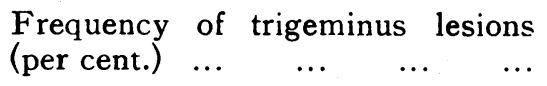 & $71^{\circ} 0$ & $70^{\circ} 0$ & $68^{\circ} 0$ \\
\hline
\end{tabular}

It appears plainly from Table II that the purely ophthalmological complaints constitute a small percentage of the first symptoms, while the trigeminus lesions are in great majority. But at the second stage of the analysis ( 4 or 5 months before the diagnosis is made) the ophthalmological symptoms have increased very considerably in number, being now found in half of all the cases, whereas the percentage of trigeminus lesions has hardly changed. The increase in the frequency of ophthalmological symptoms continues - though less abruptly so-up to the point of time at which the diagnosis is made, comprising by that time 75.2 per cent. of the cases. They are then more frequent than the trigeminus lesions, which remain at a stationary frequency level (about 70 per cent.).

It is evident from this observation not only that ophthalmological symptoms are present to a considerable extent in cases of malignant nasopharyngeal tumour, but also that these symptoms increase in frequency chiefly within the period in which the exact diagnosis has not yet been made, and in which it should thus be possible to have these cases correctly diagnosed by ophthalmological intervention.

I shall now pass on to an account of the nature of the ophthalmoneurological symptoms. The very first symptom-occurring about 10 or 11 months before the diagnosis is made-is in the majority of the cases (71.0 per cent.) trigeminal neuralgia, most of ten in the 
maxillary area, and more frequently isolated than together with either nasak stenosis, metastatic cervical glands or tubal occlusion. The only ophthalmological symptom at this stage is abducens paresis, likewise more often monosymptomatic than combined with other symptoms. Half of the ophthalmo-neurological cases of my material were purely monosymptomatic, while in the other half the combination forms were rather equally distributed over metastatic cervical glands, nasal stenosis, and tubal occlusion (about 15 per cent. each).

By the time of the second analysis -4 or 5 months before the diagnosis is made-there has occurred not only a quantitative, but also a qualitative increase in the purely ophthalmological symptoms. A detailed description of the distribution will be given in connection with the findings at the fully developed stage of the disease, because there are found no essential qualitative differences between the ophthalmological symptoms of these two stages. I shall only just mention that the purely monosymptomatic ophthalmo-neurological cases are few (3 per cent.) at this stage, since forms combined with rhinological or otological symptoms and metastatic cervical glands respectively are found in 40 per cent. each of the cases.

Accordingly a patient who at this stage of the disease applies to an ophthalmologist on account of eye muscle paresis, visual disturbances, or exophthalmos, with or without attending trigeminal neuralgia, will present symptoms also from the other main groups mentioned here. When the ophthalmologist is consulted by patients suffering from abducens paresis, impairment of vision, exophthalmos, or Horner's syndrome he should always suspect a malignant nasopharyngeal tumour to be the cause. He can then strengthen or weaken his suspicion partly through palpation for metastatic cervical glands in the typical area, and partly by questioning as to the presence of rhinological or otological symptoms, possibly supplemented by an oto-rhinological special examination. The ophthalmologist can thus, through these simple examinations, contribute decisively to the making of an exact diagnosis some therapeutically valuable months earlier than is generally the case.

Of the 454 patients who displayed the fully developed pathological picture 172 , or 38 per cent., presented ophthalmo-neurological symptoms.

The nature of the symptomatology appears from Table III, which shows that $75 \cdot 2$ per cent. of the cases had ophthalmological symptoms, and that two-thirds of these patients presented trigeminus lesion as well. Correspondingly two-thirds of the 68 per cent. with trigeminus lesion presented ophthalmological symptoms as well. Four patients only had neither ophthalmological 
symptoms nor trigeminus lesion. It appears from this close connection between ophthalmological symptoms and trigeminus lesion that the tumour tissue injures preferably the area round the cavernous sinus, where-as already mentioned-these cranial nerves run in intimate relation to each other.

TABLE III.

Nature and frequency of ophthalmo-neurological symptoms in 172 patients with malignant nasopharyngeal tumours.

\begin{tabular}{|c|c|c|}
\hline \multirow{2}{*}{$\begin{array}{l}\text { Eye symptoms } \\
129 \text { patients } 75^{\circ} 2 \\
\text { per cent. }\end{array}$} & $\begin{array}{l}\text { Alone } \\
40 \mathrm{ptt} \text {. } \\
23^{\circ} 4 \\
\text { per cent. }\end{array}$ & $\begin{array}{llrr}\text { Ophthalmoplegia } & \ldots & \ldots & 22 \text { patients } \\
\text { Ophthalmoplegia }+ \text { II } & \ldots & 11 \text { patients } \\
\text { Ophthalmoplegia } & + \text { Horner } & 3 \text { patients } \\
\text { II-lesion alone... } & \ldots & \ldots & 2 \text { patients } \\
\text { Exophthalmos... } & \ldots & \ldots & 2 \text { patients }\end{array}$ \\
\hline & \multicolumn{2}{|r|}{ With V-lesion 44 patients $25^{\circ} 6$ per cent. } \\
\hline ' & \multicolumn{2}{|c|}{$\begin{array}{l}\text { With other cranial nerve-lesion, partly V. } 45 \text { patients } \\
\qquad 26^{\circ} 2 \text { per cent. }\end{array}$} \\
\hline \multirow{2}{*}{$\begin{array}{c}\text { V-lesions } 117 \\
\text { patients } 68^{\circ} 0 \text { per } \\
\text { cent. }\end{array}$} & \multicolumn{2}{|c|}{ With eye symptoms 78 patients $45^{\circ} 4$ per cent. } \\
\hline & \multicolumn{2}{|c|}{ Without eye symptoms 39 patients $22^{\circ} 6$ per cent. } \\
\hline
\end{tabular}

The cases with ophthalmological symptoms can be divided in three almost equally large groups, namely (1) purely ophthalmological cases, (2) forms combined with trigeminus lesions alone, and (3) forms combined with trigeminus lesions as well as other cranial nerve lesions, most often lesions of the ninth and tenth nerves.

The frequency and nature of the individual ophthalmological symptoms proved to be fairly alike for each of these three groups. The symptoms were of the following nature, mentioned in decreasing order of frequency: Abducens paresis, paresis of the third nerve, visual pathway lesion, paresis of the fourth nerve, Horner's syndrome, and exophthalmos.

All the nerve lesions mentioned here can occur as isolated phenomena; but most often they are found in different forms of combination, for instance, as regards the ophthalmological symptoms, in varying forms of ophthalmoplegia with or without attending visual pathway lesion, Horner's syndrome, or exophthalmos. 
A direct impression of the frequency of the different cranial nerve lesions is obtained from Table IV, into which have been entered the 461 single nerve lesions presented by the 172 patients.

TABLE IV.

Nature and frequency of 461 single nerve lesions in 172 patients with malignant nasopharyngeal tumours.

\begin{tabular}{|c|c|c|c|c|c|c|c|c|}
\hline \multicolumn{2}{|c|}{ Nerve lesions } & VI & III & IV & II & Horner & V & Others \\
\hline \multirow[t]{2}{*}{ Frequency } & Abs. & 1.14 & 42 & 29 & 29 & 27 & 119 & 101 \\
\hline & per cent. & $24^{\circ} 0$ & $9^{*} 1$ & $6 \cdot 3$ & $6 \cdot 3$ & 58 & $25^{\circ} 7$ & $21^{\circ} 8$ \\
\hline
\end{tabular}

It appears' from this table that eye nerve lesions constitute more than half of the total number of cranial nerve lesions, partly in the form of ophthalmoplegias (39.4 per cent.), partly as visual pathway lesions (6.3 per cent.), and Horner's syndrome ( $5 \cdot 8$ per cent.). Among the ophthalmoplegias the predominating group is that of paresis of the sixth cranial nerve, which constitutes one-fourth of the total number of nerve lesions, being thus almost equal in frequency to trigeminus lesions, which comprise 25.7 per cent.

Pareses of the third and fourth cranial nerves are of much rarer occurrence. The same is the case with pareses of the other cranial nerves (VII, and IX-XII), which .altogether constitute $21 \cdot 8$ per cent. distributed over 7 cranial nerves, chiefly the bulbar nerves.

Of the 461 cranial nerve lesions 6.7 per cent. were bilateral lesions, which in practically all cases affected the eye nerves. Thus one-third of the optic tract lesions and one-tenth of the ophthalmoplegias were bilateral.

Thus it is plain to see that the ophthalmological symptoms play a predominant part in the symptomatology.

The ophthalmoplegias generally manifested themselves as massive paralyses with associated clinical findings. The visual pathway lesions, on the other hand, presented various degrees of severity from a slight impairment of vision to the more frequent total amaurosis with atrophy of the optic nerve or choked disc. Quadrantanopsia or hemianopsia were of rarer occurrence.

Exophthalmos due to penetration of the tumour into the orbit was found in 19 patients with varying degrees of chemosis and disturbances of motility, usually associated with a poor general state.

I shall not go into details with regard to the trigeminus lesions, but only just mention that they consist most frequently in 
neuralgias in one or more of the three branches, whereas the motor root is more rarely affected.

As mentioned before, we have to do with multiple cranial nerve syndromes in a great number of the cases; but it would be going too far to go into further details regarding the nature of these syndromes. Yet I want to mention, by way of example, one cranial nerve syndrome with a special pathogenesis, which has not been described before, and the occurrence of which must be regarded as pathognomonic of malignant nasopharyngeal tumours.

This syndrome comprises ophthalmoplegia (most often sixth nerve paresis) and paresis of the twelfth cranial nerve and in the majority of the cases also trigeminal neuralgia. It was observed in 9 cases, so there is reason to suppose that it was not an accidental combination. The pathogenesis of the ophthalmoplegia and the trigeminal neuralgia is the usual result of tumour invasion round the cavernous sinus. The explanation of the paresis of the twelfth cranial nerve, which was present without lesion of other distal cranial nerves (so that diffuse tumour growth can be left out of account) is obtained from the lymphatic conditions. Metastatic cervical glands were observed in all these cases, and the primary tumour was found to be lateralised more often than usually, so that the lymph ran to the retropharyngeal lymph glands, which lie off the hypoglossal canal, where it compressed the nerve.

This cranial nerve syndrome is thus conditioned partly by the intra-cranial growth of the primary tumour, and partly by lymphogenous metastases, and is therefore pathognomic of a malignant neoplasm in the nasopharynx.

\section{Roentgenography}

Before passing on to the final conclusion I shall briefly state some facts regarding the roentgenography and the treatment in cases of malignant nasopharyngeal tumour.

Roentgenography may, through an ordinary lateral picture of the skull, accidentally disclose a tumour in the nasopharynx, since such a tumour will cause a characteristic deformation of the contour of the wall. More important is, however, the knowledge one gets through a basial picture for an estimate of the extent of a possible osseous destruction. Osseous destruction in the base of the skull with typical localisation in the foramen lacerum area was found in 54.3 per cent. of the ophthalmo-neurological cases. There is an intimate correlation between the roentgenological and the ophthalmo-neurological findings. It may seem an extraordinary thing that nearly half of the ophthalmo-neurological cases presented no osseous destructions, but this is due partly to the fact that the nerve lesions were exocranial, and partly to the fact that the primary tumour can invade the base of the skull through the already existing foramina. 


\section{Treatment}

The treatment of malignant nasopharyngeal tumours is now purely radiological, as there is contraindication for operative treatment (both from a technical and from a biological point of view) of such deep-seated and invading tumour forms. Fortunately such tumours are generally rather radiosensitive on account of their biologically immature character.

A strong, external, protracted, fractional X-ray or teleradium treatment is given, which on rare occasions is supplemented by a local radium or $\mathrm{X}$-ray treatment. The $\mathrm{X}$-ray treatment is given in anterior and lateral fields (Fig. 16) in doses of from 4,000 to 10,000 r.

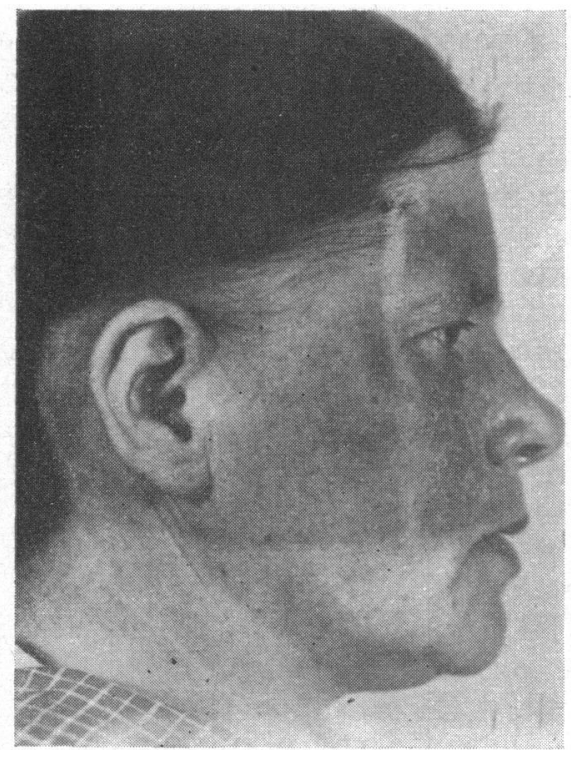

FIG. 16.

A female, aged 41 years, with characteristic pigmentation of skin portals (lateral and anterior) by the end of an X-ray treatment (9000 r skin doses). Clinically there was found a malignant nasopharyngeal tumour with extensive destruction in the base of the skull, paralysis of the sixth nerve, and Horner's syndrome; no metastatic cervical glands. Symptom-free through tbree years of observation (case 103).

through from 3 to 8 weeks. This treatment can secure a lasting freedom from symptoms (over 5 years), but whether it does so depends to a certain extent on the histopathological type of the primary tumour and on the extent of the disease. The radiosensitive reticulum-cell sarcomas, for instance, show a " five year cure rate" of nearly 40 per cent., while the corresponding figure for the total number of cases amounts to $22 \cdot 2$ per cent. 
The prognosis is reduced for higher degrees of intracranial invasion; but it should be emphasised that even in cases of cranial nerve paresis and osseous destruction of the base, where all other treatment is in vain, it is possible to secure a lasting freedom from symptoms (over 5 years) by irradiation.

Four-fifths of the patients experience a primary improvement after irradiation; but the lasting results are confounded by a pronounced tendency to recurrence and generalisation, so accordingly the lethality is very high.

\section{Summary and conclusions}

Finally I shall sum up what I have said about nasopharyngeal tumours.

The malignant nasopharyngeal tumours are fairly rare tumour forms, but not so rare as they were previously supposed to be. They occur in all age-classes, and are more frequent among men than among women.

The symptomatology, which is very polymorphous and, therefore, to a great extent gives occasion to mistaken diagnoses and wrong treatment can be divided schematically in four groups : (1) Ophthalmo-neurological symptoms due to penetration of the tumour into the base of the skull and the parapharyngeal space, (2) local rhinological, (3) otological symptoms due to the exophytic growth of the primary tumour, and finally (4) metastatic cervical glands, which are typically localised and early developed.

A fractional analysis of the symptomatology at different stages of the disease showed that the ophthalmo-neurological symptoms occur both earlier and to a greater extent than is generally known, and that the ophthalmological symptoms predominate, chiefly as ophthalmoplegias with or without attending trigeminal neuralgias.

Patients suffering from unrecognised, malignant, nasopharyngeal tumours will therefore often apply first to an ophthalmologist on account of ophthalmoplegia or impairment of vision. The ophthalmologist can then do good service in making the exact diagnosis. To do so he must be on his guard with all ophthalmoplegias and visual impairments, etc., of unknown aetiology. The suspicion of a malignant nasopharyngeal tumour is strengthened or weakened partly through palpation for metastatic cervical glands in the typical area posterior to the angle of the man dible, and partly through oto-rhinological special examinations.

If these lines are followed a great number of otherwise mistakenly diagnosed cases will become recognised several important months earlier than is generally the case, and the adequate irradiation treatment will then be instituted at an earlier and, for the results of treatment, more favourable point of time. 
The effect of modern intensified irradiation, which is often surprising in the cases of these immature tumour forms, makes the proper background for a more extensive ophthalmo-neurological diagnostication along the lines indicated here, which, when followed, will benefit the patients and reflect great credit on the doctors.

\section{REFERENCES}

GODTFREDSEN, ERIK.-Ophthalmological and neurological symptoms in malignant nasopharyngeal tumours. A clinical study comprising 454 cases. With special reference to histopathology and the possibility of earlier recognition. Acta Ophthal., Suppl. XXII, 1944; Acta Psych. and Neurol., Suppl. XXXIV, 1944 ; Acta Oto-Laryngol., Suppl. LIX, 1944 ; Acta Path and Microbiol. Sc., Suppl. LV, 1944 ; Diss., p. 320, 53 Fig., Munksgaard, Copenhagen, 1944.

\section{SOME RESEARCHES ON THE RESPIRATION OF THE CORNEA IN ALBINO RATS* $\dagger$}

BY

\section{A. BAKKER \\ GRONINGEN \\ Introduction}

IN studying literature dealing with the respiration of the cornea, we get the impression that our knowledge concerning this subject is rather defective. At any rate, we may say that the question whether the cornea uses the oxygen of the surrounding air is still far from settled.

Duke-Elder ${ }^{1}$ assumes that the cornea possesses a respiratory mechanism, whereby gases are actively transpired through the agency of the epithelium and the endothelium.

Bullot ${ }^{2}$, arguing on the grounds that in his experiments the endothelium of rabbits' eyes dies when the surrounding air is replaced by a mixture of 1 part air and 14 parts hydrogen, is of the opinion that the endothelium needs the oxygen from the surrounding air. This investigator placed the entire enucleated eyeball of the rabbit, with its epithelium scraped off, in an atmosphere of moist air at $35^{\circ} \mathrm{C}$. and observed that, after 15 hours, the endothelium was still living over the entire surface. When, however, the surrounding air was sufficiently rarefied with hydrogen (e.g., $1 / 15)$ it was established that after the same length of time all the endothelium cells were dead.

* From the Histological Institute, University Groningen, Director: Professor J. de Haan, M.D. † Received for publication, December 7, 1946. 